Part of Journal of Research of the National Bureau of Standards, Volume 35, December 1945

\title{
ASSEMBLY AND CALIBRATION OF A DENSITY BALANCE FOR LIQUID HYDROCARBONS ${ }^{1}$
}

\author{
By Alphonse F. Forziati, ${ }^{2}$ Beveridge J. Mair, and Frederick D. Rossini
}

\section{ABSTRACT}

The assembly and calibration are described of a density balance for rapidly measuring the densities of liquid hydrocarbons on samples as small as 9 milliliters in volume. The reproducibility of the measurements is \pm 0.00002 to \pm 0.00003 gram per milliliter and the over-all uncertainty is estimated to be about \pm 0.00005 gram per milliliter.

\section{CONTENTS}

Page

II. Apparatus

III. Procedure _._. 516

IV. Calibration

V. Discussion

\section{INTRODUCTION}

In connection with the work of this laboratory on the analysis, purification, and properties of hydrocarbons, it has been necessary to make measurements of the density of purified liquid hydrocarbons, and of mixtures of hydrocarbons, on samples as small as $9 \mathrm{ml}$ in volume, rapidly and with a precision of several parts per 100,000. This report describes the assembly and calibration of a density balance for such measurements.

\section{APPARATUS}

The assembly of the density balance is shown in figure 1, with the detailed parts described in the legends.

A diagram of the controls for the density balance is shown in figure 2 , with details given in the legend.

The scale on this particular balance ranges ${ }^{3}$ from 0.65 to $0.95 \mathrm{~g} / \mathrm{ml}$, with the smallest division on the chainomatic scale corresponding to $0.0001 \mathrm{~g} / \mathrm{ml}$ and readings with the vernier being made to 0.00001 $\mathrm{g} / \mathrm{ml}$.

For containing the samples to be measured, test tubes having an inside diameter of $14 \mathrm{~mm}$ and an inside depth of $138 \mathrm{~mm}$ were se-

1 This investigation was performed at the National Bureau of Standards as part of the work of the American Petroleum Institute Research Project 6 on the "Analysis, purification, and properties of hydrocarbons."

${ }_{2}$ Research Associate on the American Petroleum Institute Research Project 6 at the National Bureau of Standards.

3 Other ranges of scale readings may be obtained as required. 


\section{Journal of Research of the National Bureau of Standards}

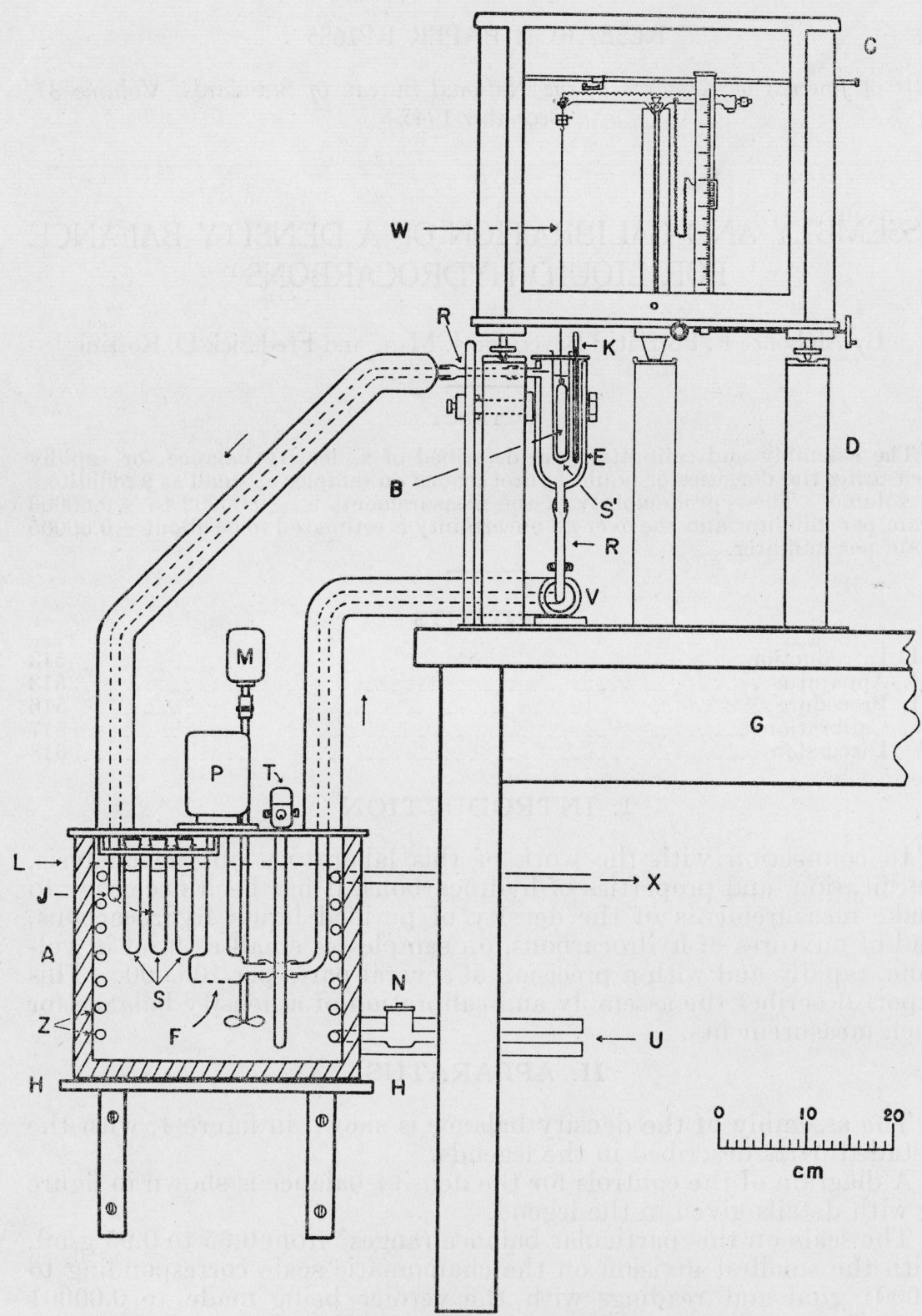

Figure 1.-Assembly of density balance.

$A$, Constant-temperature water reservoir; $B$, bob or plummet (volume $5 \mathrm{ml}$ ); $C$, chainomatic balance (see text); $D$, metal supporting stand; $E$, vacuum-jacketed glass water bath; $F$, water inlet to pump; $G$, table; $H$, shelf (fastened to wall); $J$, water return from $E ; K$, spt cial mercury-in-glass thermometer (graduated from 19.90 to 20.10 , or from 24.90 to 25.10 , or from 29.90 to 30.10 , with a sensitivity on the scale of $1 \mathrm{~mm}$ equal to $0.05^{\circ} \mathrm{C}$ ); $L$, level of water; $M$, motor for stirrer; $N$, solenoid valve; $P$, water pump; $R$, connection of rubber tubing; $S$, $S^{\prime}$, test tubes for containing samples under observation; $T$, mercury-type thermoregurubber tubing; $S$, $S^{\prime}$, test tubes for containing samples under observation; $T$, mercury-type thermoregu-
lator; $U$, inlet for cooling water; $V$, valve for controlling flow of water through $E$; $W$, platinum wire, 0.003 inch in diameter; $\boldsymbol{X}$, outlet for cooling water; $\boldsymbol{Z}$, coil for cooling water. 


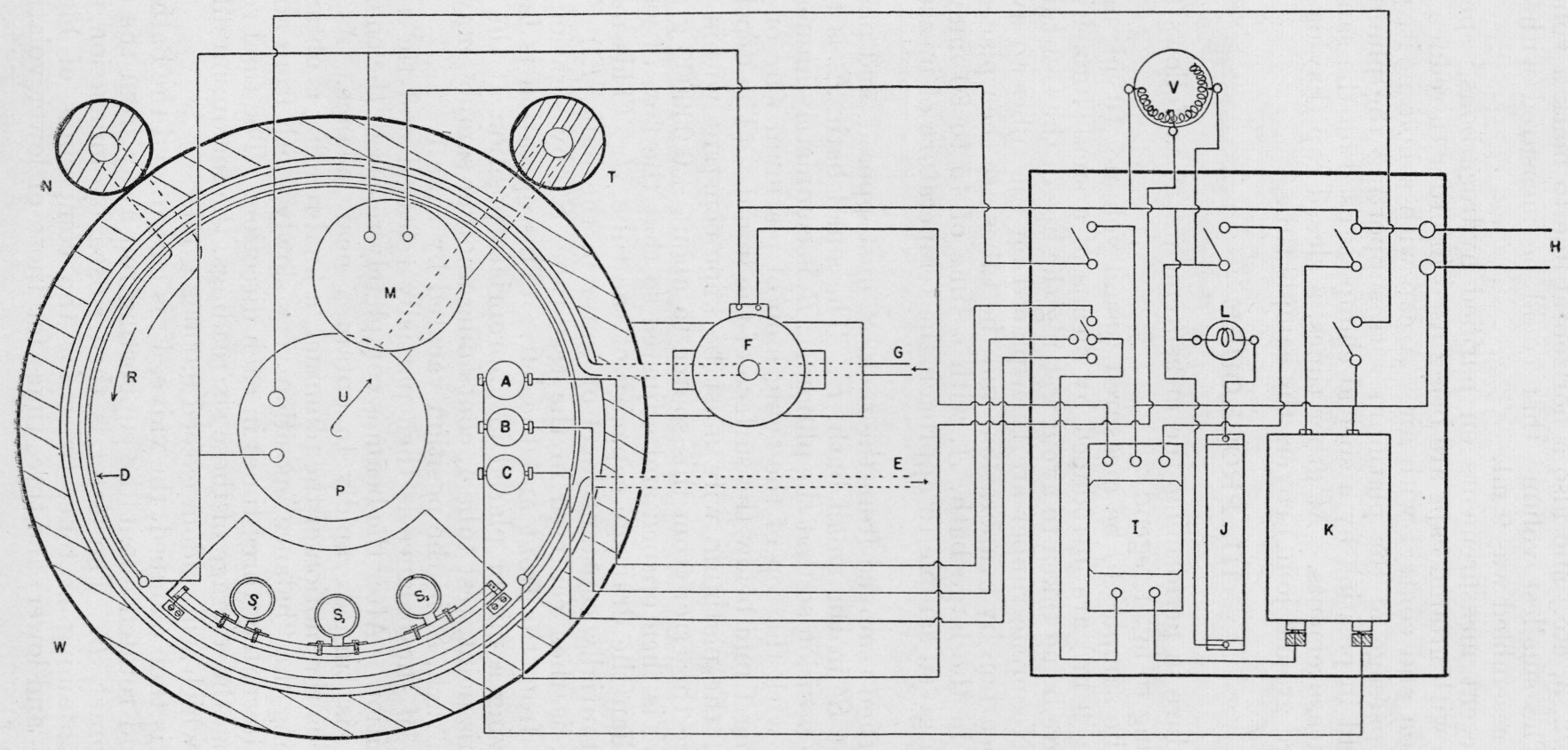

Figure 2.-Diagram of controls of the density balance.

$A, B, C$, Mercury-type thermoregulators, set to control at $20^{\circ}, 25^{\circ}$, and $30^{\circ} \mathrm{C}$, respectiv ly, with a maximum amplitude in the control cycle of about $0.005^{\circ} \mathrm{C} ; D, 100$ watt heater in copper tube, $L$, discharge for water, $F$, solenoid valve (\%/-in. iron-pipe connections); $G$, inlet for cooling water, $H$, electric-power line, 110 -volt alternating current, 1 , relay (midget), $J, 1,000$-ohm resistance (10watts); $K$, rectilying transormer, 110-volt alternating current to 6 volts, direct current; 1 , miniature lamp, 14 volts $M$, stirrer motor; $N$, return for water; $P$, circulating water pump; $R$, water from $N ; S_{1}, S_{2}$, $S_{3}$, test tubes containing samples for observation, $T$, thermally insulated pipe connecting large water reservoir and the vacuum-jacketed glass water bath; $U$, path of water to $T$; $V$, variable transformer, 115 volts aternating current, 100 watts; $W$ constant-temperature water reservoir. 
lected from standard Pyrex glass stock. On each tube was engraved a number $(1,2,3$, etc.) and also a horizontal line to mark a volume of $10 \mathrm{ml}$. The smallest volume that could be measured with the apparatus as assembled was $9 \mathrm{ml}$.

In more recent measurements on purified hydrocarbons, ${ }^{4}$ special sample tubes with ground caps are being used in order to reduce loss by evaporation and contact with air. A cap with a hole in it, permitting free passage of the platinum wire supporting the plummet and being held in position by a special clamp, is used on the sample during the measurements. At other times, a closed cap (having the same standard ground joint) covers the sample tube.

\section{PROCEDURE}

The procedure in performing the measurements is as follows (the letters referring to fig. 1 legend):

Three of the samples to be measured (usual volume, $10 \mathrm{ml}$; minimum volume, $9 \mathrm{ml}$ ) are introduced by means of a small funnel into test tubes, care being taken to avoid wetting the lips of the test tubes. Stoppers of Neoprene rubber are then placed on the tubes to avoid loss of hydrocarbon by evaporation, and the tubes are then placed in the rack, $S$, in the large bath, $A$, with a time of 15 to 20 minutes allowed for a given sample to approach the temperature of measurement.

Tube 1 is then removed from the rack, $S$, unstoppered, and placed in position at $S^{\prime}$ in the small bath, $E$. The small bath, $E$, is then elevated into position so that the plummet, $B$, is completely immersed in the liquid with the top of the tying loop of platinum wire on the plummet being $1 \mathrm{~mm}$ below the surface of the liquid. (The depth of immersion of the platinum wire must be kept constant within \pm 1 $\mathrm{mm}$ to reduce the error from this source to about $\pm 0.000005 \mathrm{~g} / \mathrm{ml}$.) The valve, $V$, is then opened and adjusted so that the flow of water is just less than the drainage capacity of the tube, $J$. (This maintains a substantially constant level of water in the bath, E.) The plummet, $B$, is then centered in the test tube, $S^{\prime}$, by sighting, at right angles, from the front and the left. (This operation is facilitated by having a mirror placed in appropriate position at the left side.) At this point, test tube 4, containing another sample, may be placed in the rack, $S$, in the position vacated by tube 1 .

The beam of the balance is then partially released and the rider placed in position. After the beam is completely released, the chainomatic crank is turned rapidly to obtain a near balance. As the liquid dampens the motion of the plummet, a final reading is obtained by approaching the "balance" position very slowly, both from above and below, alternately three times in each direction. The final reading is taken as the average of these six readings, the maximum difference among which will seldom exceed $0.00002 \mathrm{~g} / \mathrm{ml}$.

The beam is then clamped, the valve, $V$, is closed, and the bath, $E$, is lowered and rotated about the supporting rod away from the suspended plummet, $B$. The latter is then rinsed by immersion in a test tube containing acetone, twice for the compounds of higher volatility $\left(\mathrm{C}_{7}\right.$ and lower $)$ and three times for those of lower volatility $\left(\mathrm{C}_{8}\right.$ and higher).

A. F. Forziati and F. D. Rossini, National Bureau of Standards. American Petroleum Institute Research Project 6. Unpublished data. 
A period of 10 minutes is allowed for the plummet to become dry before immersing it in the next sample. During this interval, test tube 1 is removed from the small bath, $E$, the outside of the test tube is dried with a clean cloth, the sample is transferred to its original container, and the tube is cleaned by means of several rinsings with acetone and placed on the suction rack for drying.

At the beginning, middle, and end of each series of measurements, one of the standard reference liquids (2,2,4-trimethylpentane for paraffins, methylcyclohexane for naphthenes, and toluene for aromatics) is included among the samples measured in order to check the calibration of the balance (see following section).

The above procedure, which requires about 20 minutes per sample in steady operation, is that followed for the precise measurements on purified hydrocarbons. For routine analytical measurements (to $\pm 0.0001 \mathrm{~g} / \mathrm{ml}$ ) on a series of fractions of distillate, considerable time is saved by eliminating some of the above steps; reducing the time of waiting for temperature equilibrium, eliminating some of the calibrations, etc. The abbreviated procedure requires about 10 minutes per sample in steady operation.

\section{CALIBRATION}

The balance was calibrated for measurements of density by making observations on a series of seven hydrocarbons (including the three NBS Standard Samples of hydrocarbons, which are certified with respect to values of density, namely, 2,2,4-trimethylpentane, No. 217; methylcyclohexane, No. 218; and toluene, No. 211 a), for which values of density were determined ${ }^{5}$ under the supervision of $\mathrm{E}$. L. Peffer in the Bureau's Capacity and Density Section, for air-saturated material, at $20^{\circ}, 25^{\circ}$, and $30^{\circ} \mathrm{C}$, with an estimated over-all uncertainty of $\pm 0.00002 \mathrm{~g} / \mathrm{ml}$. In reducing the scale readings, corrections (from zero to $0.00003 \mathrm{~g} / \mathrm{ml}$ ) were made for slight inequalities in the spacing of the notches on the beam, which inequalities were determined by measurements with known masses hanging from the left arm of the beam as the rider on the beam was moved successively from one notch to another.

The results of the observations on the calibrating samples are given in figure 3 , which gives on the scale of abscissas the scale reading and on the scale of ordinates the scale reading less the certified value of density, in $10^{-5} \mathrm{~g} / \mathrm{ml}$. For the measurements, the balance was adjusted so that, for 2,2,4-trimethylpentane (NBS Standard Sample 217 ) at $25^{\circ} \mathrm{C}$, the scale reading was identical with the certified value for this temperature. Linear calibrating lines were drawn for each of the three temperatures, as shown, with the line for $25^{\circ} \mathrm{C}$ being made to go through zero at the density for the Standard Sample of 2,2,4-trimethylpentane.

Table 1 summarizes departure of the observed points in figure 3 from the appropriate calibrating line. It is seen that the maximum departure is $\pm 0.00003 \mathrm{~g} / \mathrm{ml}$ and that the average departures are $\pm 0.00002 \mathrm{~g} / \mathrm{ml}$.

\footnotetext{
8 The method is described in Tech. Pap. BS T77(1916) and Bul. BS 9, 405 (1913) S197.
} 


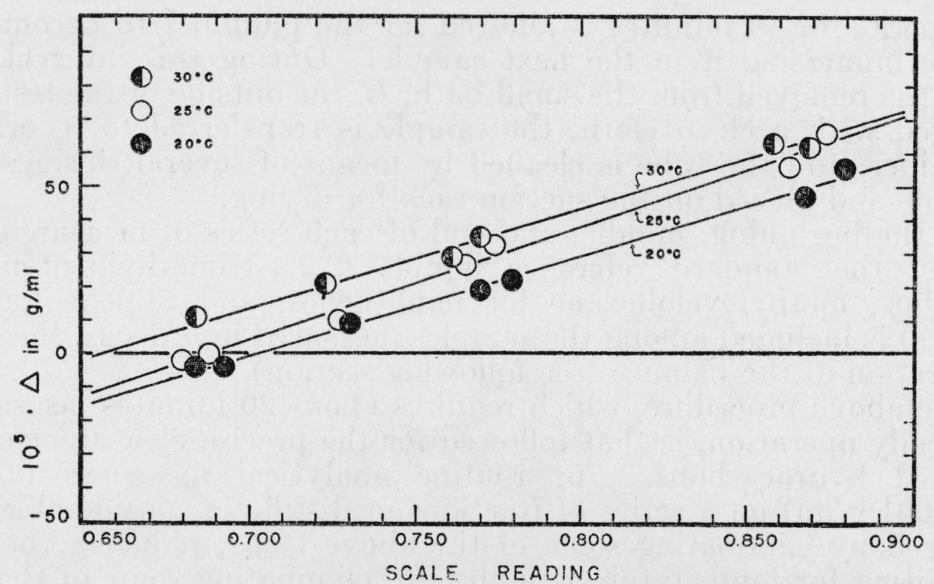

FIGURE 3.-Calibrating data for the density balance.

The scale of ordinate gives $10^{5}$ times the value of the difference, scale reading less the value of density certified for the given hydrocarbon, at the given temperature. The scale of abscissas gives the scale reading. The three sets of measurements apply to $20^{\circ}, 25^{\circ}$, and $30^{\circ} \mathrm{C}$, as indicated.

TABLE 1.-Departures of the observations on the certified samples from the respective calibrating lines

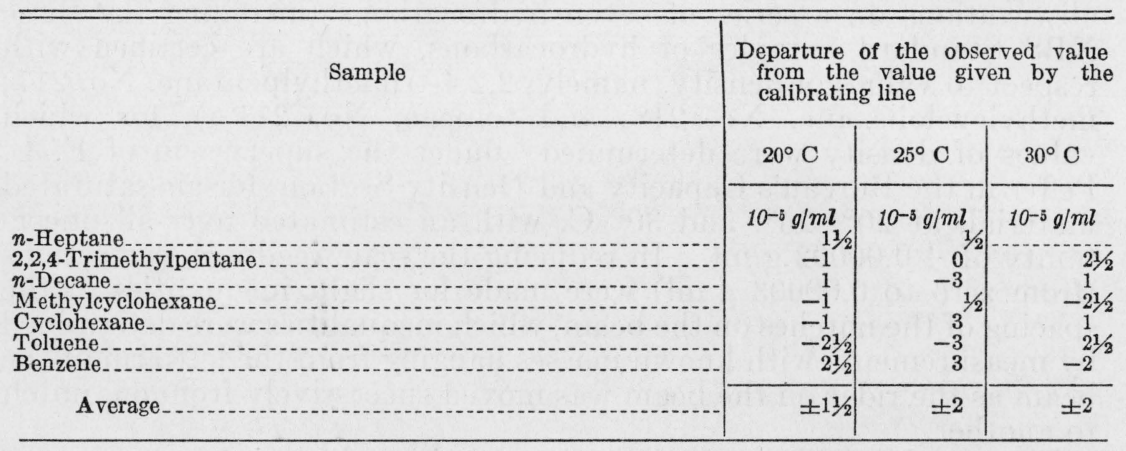

\section{DISCUSSION}

As the density of liquid hydrocarbons changes about $0.00001 \mathrm{~g} / \mathrm{ml}$ for a change in temperature of $0.01 \mathrm{deg} \mathrm{C}$, and as the sensitivity of the balance is $0.00001 \mathrm{~g} / \mathrm{ml}$, changes in temperature of the sample under observation in excess of $0.01 \mathrm{deg}$ will be reflected as changes in the scale readings at balance. For precise work, it is desirable to have a thermometric control that produces a maximum variation in the temperature of the sample of not more than $0.01 \mathrm{deg}$. In the apparatus assembled in this laboratory, the thermometric control produced temperatures having a maximum variation of about 0.005 deg. The absolute value of the temperature of the measurements made at the three controlled temperatures was determined at appropriate intervals by placing a platinum resistance thermometer $(25$ ohms) in place of the plummet and determining the resistance of the platinum thermometer with a Mueller bridge. The same observations yielded the magnitude of the variation of the temperature. 
The effect, on this method of measuring density, of differences in surface tension of the samples under investigation, with resulting different forces on the thin platinum wire supporting the plummet, was calculated to be negligible. Because the surface tension of hydrocarbons varies in a more or less regular manner with density, the main effect of differences in surface tension in going from hydrocarbons of low density to those of high density becomes incorporated as part of the calibration correction. The net effect of surface tension then reduces to the effect of the difference in surface tension for hydrocarbons of the same or nearly the same density. Examination of the available data indicates that, in the range of density 0.60 to 0.90 , hydrocarbons of the same or nearly the same density have values of surface tension that differ by not more than about one dyne $/ \mathrm{cm}$. This would correspond to a maximum error of 0.000005 $\mathrm{gm} / \mathrm{ml}$ in density.

The effect, on the density, of absorption by the hydrocarbon of water vapor from the air was determined by measuring the difference between the density of a sample dried by filtration through silica gel and of this same sample subsequently saturated with water. The observed differences, density of sample saturated with water less the density of the dried sample, were $0.00001,-0.00001$, and -0.00003 $\mathrm{g} / \mathrm{ml}$ for 2,2,4-trimethylpentane, methylcyclohexane, and toluene, respectively. During the measurements on the material saturated with water, a globule of water was permitted to lie at the bottom of the tube containing the hydrocarbon. With normal precautions, it appears that errors from this source are well within the limits of uncertainty.

In order to ascertain whether the measurements as made are for hydrocarbon saturated with air, the difference was determined between the density of a freshly distilled sample and of this same sample subsequently saturated with air. The observed differences, density of freshly distilled sample less that of the air-saturated sample, were $-0.00001,0.00001$, and $0.00001 \mathrm{~g} / \mathrm{ml}$ for $2,2,4$-trimethylpentane, methylcyclohexane, and toluene, respectively. It appears that measurements made by the present procedure may be ascribed, well within the limits of uncertainty, to hydrocarbon material saturated with air.

From the results of repeated observations on various samples, the reproducibility of the observations made with this density balance is estimated to be \pm 0.00002 to $\pm 0.00003 \mathrm{~g} / \mathrm{ml}$. From the data given in table 1 and figure 3, the over-all uncertainty of the measurements of density of a given sample is estimated to be about $\pm 0.00005 \mathrm{~g} / \mathrm{ml}$.

The authors are indebted to E. L. Peffer, chief of the Bureau's Capacity and Density Section, for providing the absolute values of the densities of the reference and Standard Samples used in the calibrations reported in this paper.

WASHINGTON, June 1, 1945. 